\title{
Reframing the barrier: Nurse-led, integrated palliative care program in the context of HIV in the United States
}

\author{
Samantha R McGraw ${ }^{1 *}$, Rebecca A Gass ${ }^{2}$, Jean FitzGibbons BA ${ }^{1}$, Cindy Magrini ${ }^{1}$, Stuart Fisk ${ }^{1}$ and Randy S Hebert ${ }^{2}$ \\ ${ }^{1}$ Positive Health Clinic, Allegheny Health Network, 1307 Federal St Suite B110, Pittsburgh, PA 15212, USA \\ ${ }^{2}$ Division of Palliative Medicine, Department of Medicine, Allegheny Health Network, 4800 Friendship Avenue, Pittsburgh, PA 15212, USA
}

\begin{abstract}
Symptom burden of patients infected with HIV remains prevalent, despite providers' attempts to address palliative needs. Lack of access to pain management experts is described as a barrier to effective palliative care. We integrated an advance certified hospice palliative care nurse practitioner into an outpatient, inner-city clinic serving the comprehensive needs of ambulatory patients infected with HIV. Patients' symptom burden was assessed with the Brief Pain Inventory (BPI) and M.D. Anderson's Brief Pain Inventory and Symptom Inventory Scale (MDASI). Fourteen patients completed BPI and MDASI surveys during the initial and second visits. Paired sample t-tests and exact sign tests reported significant decreases in select item scores. The severity of pain, numbness and tingling, and sadness improved from first to second visit. Patients also reported less symptom interference on general activity, overall mood, and enjoyment of life. Bivariate correlations showed a significant relationship between select item scores and length of time between first and second visits (e.g., severity of pain, fatigue, shortness of breath, and lack of appetite as reported on the MDASI), suggesting length of time after initial visit contributed to the difference in certain item scores. Our study serves as a building block for future studies to examine our model and test the efficacy through more stringent methods. We believe our model addresses barriers to effective palliative care and, ultimately, reduces prevalent symptom burden in patients infected with HIV in the United States.
\end{abstract}

\section{Introduction}

The development of highly active antiretroviral therapy in the 1990's altered the course of HIV from an acute illness with high mortality to a chronic illness with a near-normal life expectancy [1]. As a result, more patients now suffer from chronic pain and other debilitating symptoms. Palliative care improves quality of life for these patients. The palliative approach focuses on symptom control to reduce the burden posed by pain and other symptoms. Despite provider engagement, prior literature suggests high symptom burden remains prevalent [2].

A significant barrier to effective palliative care is the lack of access to pain management experts [3]. We examined this barrier reframed as an integrated program led by trained providers. Few examples exist in the literature describing integrated palliative care clinics in the context of HIV [4,5]. One such study [6] found palliative care delivered by trained clinicians in this setting significantly improved physical, mental, and overall health. Another study [7] evaluated a similar program led by trained nurses. Results of this study [7] indicated pain was not significantly improved. Until recently, prior studies [6,7] concerning HIV-infected patients examined integrated palliative care in developing countries due to the high disease burden in these areas. Perry [8] published the first study describing high symptom burden among HIV-infected patients located in the U.S., but did not address symptom improvement.

Given the gap in literature and the need for improved symptom burden, we explored the results of our palliative care program embedded in an outpatient HIV clinic. Our main focus was on improving symptom burden. Prior literature [9] suggested effective symptom control strengthens treatment compliance and increases life expectancy for patients infected with HIV.
We approached the results of this collaboration through inductive reasoning and did not generate specific hypotheses. However, we expected to improve symptom burden on some level.

\section{Methods}

\section{Setting}

Collaboration between the Positive Health Clinic (PHC) and the Division of Palliative Medicine was the result of combining a growing need within the PHC to improve symptom-directed care with the need and capabilities of the Division of Palliative Medicine to broaden the scope of services to outpatient, palliative medicine.

The PHC is an inner-city, HIV primary care clinic which receives funding under the federal Ryan White Care Act to provide outpatient, ambulatory care, mental health services, and medical case management to almost $800 \mathrm{HIV}$-positive persons living in the Pittsburgh, Pennsylvania area. The clinic is staffed by two infectious disease physicians, an internist, a family medicine physician, a pharmacist, two HIV nurse practitioners, and an HIV physician assistant. Additional support staff includes nurses and medical assistants, social workers, a mental health therapist, a psychiatrist and a patient advocate. The $\mathrm{PHC}$ focuses on engaging patients in active, continuing medical care

Correspondence to: Samantha R. McGraw, BS, Positive Health Clinic, Allegheny Health Network, 1307 Federal St, Ste B110, Pittsburgh, PA 15212, USA, Tel: 412 359-6468. E-mail: Samantha.MCGRAW@ahn.org

Key words: barriers, human immunodeficiency virus (HIV) therapy, integrated outpatient clinic, pain management experts, palliative care, symptom burden

Received: June 11, 2016; Accepted: July 04, 2016; Published: July 08, 2016 
through a multidisciplinary approach, while providing individualizedlevels of support for each patient to reach medical goals and achieve high-levels of adherence to therapy.

The Division of Palliative Medicine of the Allegheny Health Network is an inpatient-based palliative medicine consultation program serving all seven hospitals of the Allegheny Health Network in Western Pennsylvania. At the time of collaboration, the Division of Palliative Medicine consisted of eight physicians and seven nurse practitioners, all of whom were board certified in hospice and palliative medicine.

In February 2013, a nurse practitioner (author RAG) with Advanced Certification in Hospice and Palliative Medicine (ACHPN ${ }^{\star}$ ) was embedded in the PHC two days a week, at 4 hours per day. In contrast to the study conducted by Lowther [7] in Kenya describing an ineffective nurse-led, palliative care program, the nurse practitioner had over ten years of experience in hospice and inpatient palliative medicine, providing for an evaluation of a more robust skill set presented by the palliative care nurse provider.

\section{Subjects}

Forty-nine patients, with complex chronic pain or comorbidities, were referred to the ACHPN during the first 16 months of collaboration. All patients were referred by a PHC physician. Of the 49 patients, 14 (28.57\%) completed symptom burden assessment surveys at both the initial visit (referred to as first visit) and a second visit with ACHPN. Only these 14 patients were included in subsequent analyses. Prior to referral, all 14 patients received palliative care by a routine HIV care provider at the PHC, for a period of no less than six months; patients were prescribed analgesics during this period.

\section{Intervention}

Palliative care visits with the ACHPN focused on symptom management and related concerns (e.g., pain, constipation, diarrhea, anxiety, and depression). The ACHPN evaluated the impact of symptom severity on patients' quality of life through open discussions. In order to improve overall symptom burden, patients were engaged in educational topics concerning coping skills, physical versus psychological pain, medication compliance, and pain management. In our study, each patient presented with chronic pain symptoms and was prescribed analgesics. The ACHPN discussed prescribed medications at length with each patient, including side effects and potential dependency. As such, the opiate policy was reviewed and a contract or agreement was signed stating the patient would comply with all medication instructions. Patients violating the contract were counselled on safety with opiates, addiction versus dependence, tolerance, and responsibility of opiates in the home. Majority of education was given verbally. However, each patient received a copy of the opiate contract and instructions detailing appropriate actions to take in the event of an overdose. A focused physical exam was also conducted as appropriate to the symptoms of the comorbidities. A portion of the visit was dedicated to discussing goals of care, benefits versus burdens of treatment options, and, when appropriate, advanced directives.

The integration model allowed our palliative care provider to address patient concerns with a multidisciplinary approach. The ACHPN consulted PHC providers and additional staff (e.g., registered nurses and social workers) to address social barriers presented by select patients, such as lack of transportation, financial concerns, and personal relationship struggles. Resolution of these barriers provided opportunities for patients to actively engage in palliative care visits, thus, increasing the viability of improved symptom burden.

\section{Measures}

Patients' symptom burden was assessed with the Brief Pain Inventory (BPI) [10] and M.D. Anderson's Brief Pain Inventory and Symptom Inventory Scale (MDASI) [11]. The short version of the BPI assesses four pain severity items and seven pain interference items (in the last 24 hours) rated on $0-10$ scales, in addition to a question about percentage of pain relief by analgesics. The MDASI assesses the severity of 13 symptoms at their worst in the last 24 hours on a $0-10$ numerical rating scale, with 0 being "not present" and 10 being "as bad as you can imagine." The MDASI also measures to what degree symptoms have interfered with six daily activities: general activity, mood, work, relations with others, walking, and enjoyment of life. Interference is rated on a 0-10 scale, with 0 being "did not interfere" and 10 being "interfered completely." The smallest difference considered clinically important for the MDASI questions ranges from 0.98-1.21 [11]. Both scales are validated and reliable for application with patients infected with HIV and other chronic illnesses [12].

\section{Data collection}

All patients were asked to complete the BPI and MDASI surveys at the first and second visits with the ACHPN. Median length of time between visits was 10 weeks (IQR = 7-19). Patients were given the surveys, by PHC staff, to complete while in the waiting room prior to each visit. For the purposes of our study, initial and second visit scores were analysed as repeated measures with statistical significance indicator set as a p-value less than .05 .

\section{Analysis}

A composite score was calculated for the five most highly-rated symptoms as reported on the MDASI (i.e., score was calculated per MDASI instructions; p.23-24) [11], allowing us to determine the impact of our integrated palliative care program on the most severe symptoms described by our patients. Paired-sample t-test analyses were used to compare symptom burden scores between first and second visits, calculating differences in means for the 5 symptom composite score and each item on the BPI and MDASI scales. In instances where the distribution of differences for item responses was found to be nonnormal using Shapiro-Wilk Test of Normality (i.e., violates pairedsample t-test assumption of an approximately normal distribution among differences) and asymmetrical using descriptive statistics (e.g., skewness and kurtosis), the exact sign test was applied, calculating differences in medians and assessing consistent changes between first and second visit item scores. As this is an exploratory/feasibility study with first and second measurement scores collected at unstandardized time-intervals, two-tailed, Pearson correlations were conducted to test the influence of time between BPI and MDASI completion on outcome measurement scores and subsequent symptom burden. All analyses were conducted using IBM's Statistical Package for Social Sciences (SPSS) version 20.0 for Windows ${ }^{\circledR}$ operating system, released in 2011.

\section{Results}

Seventy-nine percent $(n=11)$ of patients were male, $64.3 \%(n=9)$ were white, and mean age was 51.1 years $(S D=7.3)$. Patients suffered from a variety of severe medical conditions with the most common (i.e., present in at least $50.0 \%$ of patients) reasons for referral being musculoskeletal and neuropathic pain (Table 1). 


\section{5 most highly-rated symptoms composite score}

Composite scores for symptom severity as reported on the MDASI were calculated for our sample's five most highly-rated items during initial visit: pain (MDASI 1; $\mathrm{M}=9.08, \mathrm{SD}=1.62$ ), fatigue (MDASI 2), numbness and tingling (MDASI 13), disturbed sleep (MDASI 9), and sadness (MDASI 11; Table 2 and Table 3). The integrated palliative care program showed a marginally significant influence on the MDASI

Table 1. Characteristics of patients who completed pain assessments during first and second palliative medicine visit $(\mathrm{N}=14)$

\begin{tabular}{|l|c|c|}
\hline Characteristic & $\underline{\mathbf{n}}$ & $\underline{\mathbf{0}}^{\mathbf{a}}$ \\
\hline Gender & 11 & $78.6 \%$ \\
$\quad$ Male & 3 & $21.4 \%$ \\
\hline Age & & \\
$\quad<40$ & 0 & $0.0 \%$ \\
$40-49$ & 6 & $42.9 \%$ \\
$50-59$ & 5 & $35.7 \%$ \\
$60-69$ & 3 & $21.4 \%$ \\
$69<$ & 0 & $0.0 \%$ \\
\hline Ethnicity & & \\
\hline Caucasian/White & 9 & $64.3 \%$ \\
\hline African American & 5 & $35.7 \%$ \\
\hline Referral Reason & & \\
\hline Anxiety & 3 & $21.43 \%$ \\
\hline Chronic respiratory condition & 4 & $28.57 \%$ \\
\hline Depression & 4 & $28.57 \%$ \\
\hline Musculoskeletal pain & 9 & $64.29 \%$ \\
\hline Neuropathic pain & 8 & $57.14 \%$ \\
\hline
\end{tabular}

${ }^{a}$ Percent column denotes percent of total population or count for each characteristic, independently (e.g. gender, age, ethnicity, comorbidities and referral reason). Concerning comorbidities and referral reasons, " $n$ " column total and "\%" column total exceeds $100 \%$ as some patients are associated with multiple comorbidities and/or referrals.

Note: $\mathrm{N}=$ population size; $\mathrm{n}=$ sample size.

Table 2. Paired samples t-test results for first and second visit scores on MDASI measure. symptom severity composite score, such that scores decreased from first visit $(\mathrm{M}=7.18, \mathrm{SD}=2.08)$ to second visit $(\mathrm{M}=6.00, \mathrm{SD}=1.91 ; p=0.050)$.

\section{Item scores}

Select aspects of symptom severity significantly decreased as reported during the second visit, compared to the initial report at the first visit with the ACHPN (Table 2-4). Pain treatments and/or medication provided an increase in percentage of symptom relief (BPI 8). Symptoms significantly relieved included pain severity, numbness and tingling, as well as sadness (i.e., 3 of the 5 most highly-rated symptoms). Patients reported experiencing significantly less pain at the time of second visit survey completion (BPI 6) compared to first visit. Pain at its worst, 24 hours prior to survey completion (BPI 3), was reported to diminish as well, such that 9 patients reported a decrease in scores; 5 patients reported no change while 0 patients reported an increase in pain at its worst. Aside from the severity of pain, patients also reported a significant reduction in severity of numbness and tingling (MDASI 13), and sadness (MDASI 11). Results suggest our palliative medicine program provided significant relief from 3 of the 5 most highly-rated symptoms, reducing the symptom burden on patients infected with HIV and providing opportunities to engage in daily life.

Palliative medicine visits also improved interference of symptoms on select aspects of daily life (Tables 2-4), such that patients reported decreasing interference with general activity (MDASI 14), from first visit to second visit. As noted above concerning the reduction in severity of sadness, interference of symptoms on the patients' mood (MDASI 15) also decreased. Ultimately, patients experienced a reduction of symptom interference on overall enjoyment of life (MDASI 19). While some aspects of symptom burden were improved from the first visit to the second visit, others lacked statistical significance.

Length of time between first and second visit (i.e., time between collection of first and second BPI and MDASI scores) did not significantly influence the increase or decrease in composite or item scores for the majority of BPI and MDASI items (Table 5 and 6).

\begin{tabular}{|c|c|c|c|c|c|c|c|c|}
\hline \multirow[t]{2}{*}{ MDASI item } & \multirow[t]{2}{*}{ n } & \multicolumn{2}{|c|}{ First visit } & \multicolumn{2}{|c|}{$\underline{\text { Second visit }}$} & \multirow[b]{2}{*}{$\mathbf{t}$} & \multirow[b]{2}{*}{ df } & \multirow[b]{2}{*}{$\mathbf{p}$} \\
\hline & & $\mathbf{M}$ & SD & $\mathbf{M}$ & SD & & & \\
\hline 2. Severity of symptoms...your fatigue (tiredness) at its worst? & 12 & 8.25 & 1.71 & 7.67 & 1.83 & 0.83 & 11 & 0.42 \\
\hline 3. ...your nausea at its worst? & 13 & 3.31 & 3.30 & 3.23 & 3.03 & 0.07 & 12 & 0.94 \\
\hline 4. ...your disturbed sleep at its worst? & 12 & 6.08 & 3.73 & 6.08 & 2.50 & 0.00 & 11 & 1.00 \\
\hline 5. ...your feelings of being distressed (upset) at its worst? & 13 & 5.08 & 3.82 & 4.69 & 3.50 & 0.47 & 12 & 0.65 \\
\hline 6. ...your shortness of breath at its worst? & 11 & 4.36 & 3.07 & 3.64 & 3.61 & 0.90 & 10 & 0.39 \\
\hline 7. ...your problem with remembering things at its worst? & 13 & 3.46 & 2.44 & 3.46 & 2.15 & 0.00 & 12 & 1.00 \\
\hline 8. ...your problem with lack of appetite at its worst? & 13 & 3.69 & 3.33 & 4.23 & 3.14 & -0.54 & 12 & 0.60 \\
\hline 9. ...your feeling drowsy (sleepy) at its worst? & 13 & 5.23 & 3.14 & 5.85 & 3.26 & -0.64 & 12 & 0.53 \\
\hline $10 . .$. your having dry mouth at its worst? & 13 & 4.92 & 3.95 & 4.46 & 3.89 & 0.62 & 12 & 0.55 \\
\hline 11. ...your feeling sad at its worst? & 13 & 5.69 & 3.68 & 3.77 & 2.59 & 2.39 & 12 & 0.03 \\
\hline 13....numbness and tingling at its worst? & 11 & 6.91 & 3.67 & 5.18 & 3.71 & 3.41 & 10 & 0.01 \\
\hline 14. Interference of symptoms...general activity? & 13 & 6.77 & 3.49 & 5.15 & 2.51 & 2.39 & 12 & 0.03 \\
\hline $15 . . . \operatorname{mood} ?$ & 12 & 6.00 & 3.28 & 4.42 & 2.75 & 2.46 & 11 & 0.03 \\
\hline 16....work (including house work)? & 12 & 7.33 & 2.23 & 6.17 & 2.41 & 1.77 & 11 & 0.11 \\
\hline 17. ...relations with other people? & 13 & 4.77 & 3.47 & 2.77 & 2.62 & 2.13 & 12 & 0.06 \\
\hline 18. ...walking? & 13 & 6.38 & 3.23 & 6.08 & 3.55 & 0.51 & 12 & 0.62 \\
\hline 19. ...enjoyment of life? & 12 & 7.42 & 2.50 & 4.50 & 2.68 & 3.30 & 11 & 0.01 \\
\hline
\end{tabular}

Note: BPI items are paraphrased. $\mathrm{n}=$ sample size; $\mathrm{M}=$ mean; $\mathrm{SD}=$ standard deviation. Items numbered 1 and 12 are not included in the above table as items were analyzed with Nonparametric Sign Test. Items 2 through 11 are measured on 11-point Likert scale ( $0=$ not present, $10=$ as bad as you can imagine). Items 13 through 19 are measured on 11 -point Likert scale $(0=$ did not interfere, $10=$ completely interfered $)$. 
Table 3. Results of nonparametric exact sign tests of appropriate BPI and MDASI items

\begin{tabular}{|c|c|c|c|c|c|c|c|c|c|}
\hline \multirow[b]{2}{*}{ Items } & \multirow[b]{2}{*}{$\mathbf{n}$} & \multicolumn{2}{|c|}{ First visit } & \multicolumn{2}{|c|}{$\underline{\text { Second visit }}$} & \multirow[b]{2}{*}{$\begin{array}{c}\text { Negative } \\
\text { Differences }\end{array}$} & \multirow[b]{2}{*}{ Positive Differences } & \multirow[b]{2}{*}{ Ties } & \multirow[b]{2}{*}{$\begin{array}{c}\text { Exact } \\
\text { Sig. }\end{array}$} \\
\hline & & Mdn & IQR & Mdn & IQR & & & & \\
\hline $\begin{array}{l}\text { BPI } \\
\text { 3. Rate your pain in the last } 24 \text { hours, at its worst. }\end{array}$ & 14 & 9.00 & $7.00-10.00$ & 7.00 & $5.75-8.00$ & 9 & 0 & 5 & 0.004 \\
\hline $\begin{array}{l}\text { 9a. Describe the degree to which pain has interfered, in the past } \\
24 \text { hours, with your...general activity. }\end{array}$ & 14 & 7.50 & $3.75-8.00$ & 5.50 & $4.00-7.00$ & 11 & 2 & 1 & 0.022 \\
\hline $\begin{array}{l}\text { MDASI } \\
\text { 1. Severity of symptoms: your pain at its worst? }\end{array}$ & 12 & 10.00 & $8.75-10.00$ & 8.00 & $5.25-9.50$ & 6 & 0 & 6 & 0.031 \\
\hline 12. ...your vomiting at its worst? & 13 & 1.50 & $.00-3.00$ & .00 & $.00-2.00$ & 5 & 2 & 6 & 0.453 \\
\hline
\end{tabular}

Note: BPI and MDASI items are paraphrased. $\mathrm{n}=$ sample size; Mdn = median; IQR = interquartile range. Significance level is indicated under Exact Sig. column.

Table 4. Paired samples t-test results for first and second visit scores on BPI measure.

\begin{tabular}{|c|c|c|c|c|c|c|c|c|}
\hline \multirow[t]{2}{*}{ BPI item } & \multirow[t]{2}{*}{ n } & \multicolumn{2}{|c|}{ First visit } & \multicolumn{2}{|c|}{$\underline{\text { Second visit }}$} & \multirow[b]{2}{*}{$\mathbf{t}$} & \multirow[b]{2}{*}{ df } & \multirow[b]{2}{*}{$\mathbf{p}$} \\
\hline & & M & SD & $\mathbf{M}$ & SD & & & \\
\hline 1. Have you had pain, other than everyday kinds of pain, today? & 11 & 1 & .00 & .91 & .30 & 1.00 & 10 & 0.34 \\
\hline 4. Rate your pain... at its least in the last 24 hours. & 14 & 4.86 & 2.14 & 4.29 & 2.09 & 1.17 & 13 & 0.26 \\
\hline 5. ...on average. & 14 & 6.57 & 1.95 & 6.36 & 1.82 & .48 & 13 & 0.64 \\
\hline 6. ....amount of pain you have right now. & 14 & 6.57 & 2.65 & 4.93 & 2.89 & 2.52 & 13 & 0.03 \\
\hline $\begin{array}{l}\text { 8. In the last } 24 \text { hours, how much relief have pain treatments or } \\
\text { medications provided }(\%) \text { ? }\end{array}$ & 13 & 50.38 & 27.57 & 71.54 & 16.76 & -2.82 & 2 & 0.02 \\
\hline $\begin{array}{l}9 \mathrm{~b} \text {. Describe the degree to which pain has interfered, in the past } 24 \\
\text { hours, with your...mood. }\end{array}$ & 13 & 6.08 & 3.04 & 4.69 & 2.50 & 1.63 & 12 & 0.13 \\
\hline 9c. ...walking ability. & 13 & 6.38 & 3.10 & 6.08 & 2.99 & .62 & 12 & 0.55 \\
\hline 9d. ...normal work. & 12 & 7.92 & 1.68 & 6.50 & 2.58 & 2.16 & 11 & 0.05 \\
\hline 9e. ...relations with other people. & 13 & 3.85 & 3.29 & 3.46 & 2.44 & .41 & 12 & 0.69 \\
\hline 9f. ...sleep. & 12 & 6.58 & 2.94 & 5.42 & 2.47 & 1.48 & 11 & 0.17 \\
\hline 9g. ...enjoyment of life. & 13 & 5.77 & 3.35 & 5.15 & 2.27 & .77 & 12 & 0.46 \\
\hline
\end{tabular}

Note: BPI items are paraphrased. $\mathrm{n}=$ sample size; $\mathrm{M}=$ mean; $\mathrm{SD}=$ standard deviation. Items numbered 2 and 7 are not included in analysis as responses were not captured through a numerical rating scale. Items numbered 3 and 9 a are not included in the above table as items were analyzed with Nonparametric Sign Test. Items 4 through 6 are measured on 11 point Likert scale $(0=$ no pain, $10=$ pain as bad as you can imagine). Item 8 is measured on a percent-scale, where $0 \%$ is no relief and $100 \%$ is complete relief. Items $9 \mathrm{~b}$ through $9 \mathrm{~g}$ are measured on 11 point Likert scale $(0=$ does not interfere, $10=$ completely interferes $)$.

Table 5. Bivariate correlation results for first and second visit scores on BPI measure.

\begin{tabular}{|c|c|c|c|c|c|}
\hline \multirow{2}{*}{\begin{tabular}{|l|} 
BPI item number \\
Symptom severity \\
\end{tabular}} & \multirow[b]{2}{*}{$\mathbf{n}$} & \multicolumn{2}{|c|}{ Paired Diff } & \multirow[b]{2}{*}{$\mathbf{r}$} & \multirow[b]{2}{*}{$\mathbf{p}$} \\
\hline & & $\mathbf{M}$ & SD & & \\
\hline 1. & 11 & 0.09 & 0.30 & 0.16 & 0.64 \\
\hline 3. & 14 & 1.50 & 1.40 & -0.22 & 0.44 \\
\hline 4. & 14 & 0.57 & 1.83 & -0.00 & 0.99 \\
\hline 5. & 14 & 0.21 & 1.67 & -0.07 & 0.82 \\
\hline 6. & 13 & 1.64 & 2.44 & -0.19 & 0.53 \\
\hline 8. & 13 & -21.15 & 27.09 & 0.04 & 0.91 \\
\hline \multicolumn{6}{|c|}{ Symptom interference } \\
\hline $9 a$ & 14 & 0.79 & 3.47 & 0.14 & 0.63 \\
\hline $9 \mathrm{~b}$. & 13 & 1.38 & 3.07 & -0.10 & 0.75 \\
\hline 9c. & 13 & 0.31 & 1.80 & -0.25 & 0.41 \\
\hline 9d. & 12 & 1.42 & 2.27 & -0.61 & 0.03 \\
\hline 9e. & 13 & 0.38 & 3.40 & -0.15 & 0.64 \\
\hline 9f. & 12 & 1.17 & 2.72 & -0.54 & 0.07 \\
\hline 9g. & 13 & 0.62 & 2.87 & -0.12 & 0.70 \\
\hline
\end{tabular}

Note: $\mathrm{n}=$ sample size; $\mathrm{M}=$ mean; $\mathrm{SD}=$ standard deviation; $\mathrm{r}=$ correlation coefficient; $\mathrm{p}=$ significance level.

However, 4 MDASI severity item scores significantly correlated with time, such that severity of pain, fatigue, shortness of breath, and lack of appetite showed a negative relationship with length of time between first and second visit scores. Three of the four MDASI severity items were not shown to significantly decrease or increase during paired t-test analyses or exact sign tests. Severity of pain (MDASI 1) was significantly correlated with time and resulted in a statistically significant decrease between first and second visit, using the exact sign test analysis.

\section{Discussion}

To our knowledge, our study is the first to evaluate an integrated palliative care model implemented in the United States. The results of our exploratory study indicate implementation of a palliative program, led by an ACHPN, can improve symptom burden for patients infected with HIV in the U.S.

\section{Symptom severity}

For our particular sample, patients rated pain, fatigue, numbness and tingling, disturbed sleep, and sadness as the most severe symptoms experienced in the 24-hours prior to completion of the first MDASI measurement scale. These results confirm the findings of previous literature examining symptom burden in patients infected with HIV 
Table 6. Bivariate correlation results for first and second visit scores on MDASI measure.

\begin{tabular}{|c|c|c|c|c|c|}
\hline \multirow[t]{2}{*}{ MDASI item number } & \multirow[t]{2}{*}{$\mathbf{n}$} & \multicolumn{2}{|c|}{ Paired Diff } & \multirow[b]{2}{*}{$\mathbf{r}$} & \multirow[b]{2}{*}{$\mathbf{p}$} \\
\hline & & $\mathbf{M}$ & SD & & \\
\hline \multicolumn{6}{|l|}{ Symptom severity } \\
\hline 1. & 12 & 1.67 & 2.19 & -0.83 & 0.001 \\
\hline 2. & 12 & 0.58 & 2.43 & -0.72 & 0.01 \\
\hline 3. & 13 & 0.08 & 3.73 & -0.46 & 0.12 \\
\hline 4. & 12 & 0.00 & 3.41 & -0.00 & 0.98 \\
\hline 5. & 13 & 0.38 & 2.96 & -0.35 & 0.24 \\
\hline 6. & 11 & 0.73 & 2.69 & -0.61 & 0.05 \\
\hline 7. & 13 & 0.00 & 2.61 & -0.34 & 0.25 \\
\hline 8. & 13 & -0.54 & 3.62 & -0.60 & 0.03 \\
\hline 9. & 13 & -0.62 & 3.45 & -0.40 & 0.18 \\
\hline 10. & 13 & 0.46 & 2.70 & -0.23 & 0.45 \\
\hline 11. & 13 & 1.92 & 2.90 & -0.48 & 0.10 \\
\hline 12. & 13 & 0.46 & 4.16 & -0.38 & 0.20 \\
\hline 13. & 11 & 1.73 & 1.68 & -0.25 & 0.46 \\
\hline \multicolumn{6}{|l|}{ Symptom interference } \\
\hline 14. & 13 & 1.62 & 2.43 & -0.34 & 0.25 \\
\hline 15. & 12 & 1.58 & 2.23 & -0.26 & 0.41 \\
\hline 16. & 12 & 1.17 & 2.29 & -0.41 & 0.19 \\
\hline 17. & 13 & 2.00 & 3.39 & -0.34 & 0.25 \\
\hline 18. & 13 & 0.31 & 2.18 & -0.26 & 0.40 \\
\hline 19. & 12 & 2.92 & 3.06 & -0.02 & 0.95 \\
\hline
\end{tabular}

Note: $\mathrm{n}=$ sample size; $\mathrm{M}=$ mean; $\mathrm{SD}=$ standard deviation; $\mathrm{r}=$ correlation coefficient; $\mathrm{p}$ = significance level.

$[2,8]$. The severity of 3 out of 5 symptoms significantly improved after one visit with ACHPN, as reported approximately 10 weeks later. Similar results have been reported by previous studies conducted in developing countries [6,7]. Harding [6] reported a statistically significant improvement in pain severity after 10 weeks, as measure by the MOS-HIV. Physicians involved in the study were exposed to palliative care education prior to engaging with patients. Alternatively, Lowther [7] employed nurses as the primary palliative care provider, with contradictory results, such that pain severity was not significantly improved, also measured by the MOS-HIV. While the nurses in the Lowther [7] study participated in 2 weeks of full-time education and training, our nurse practitioner had extensive experience and certification in the field of palliative and hospice care. The robust skill set presented by the ACHPN could be a contributing factor in the differing results compared to Lowther [7] and the similar results compared to Harding [6]. Differences could also be attributed to cultural aspects present in developing versus developed countries (e.g., access to health insurance, funding, and relevant medication) [13]. Previously cited studies [6,7] were also more stringent concerning timeframe of outcome measurements.

For our study, the length of time between reported scores for each individual was varied. However, this relationship did not appear statistically significant across all items. For the 5 most highly-rated symptoms on the MDASI, only pain demonstrated a relationship. As all patients in our sample presented with chronic pain issues and were prescribed analgesics, the amount of time compliant with pain medication could certainly influence reported scores during the second visit (i.e., analgesics are designed to reduce pain, providing a direct link between medication and severity of pain). The severity of the remaining symptoms (e.g., numbness and tingling, and sadness) was not correlated, leading us to conclude engagement in our integrated palliative care program, which includes a pain medication component, provided a significant influence on these item scores.
Unlike the MDASI, pain severity as reported on the BPI was not correlated with time between first and second visit. Questions on the BPI and MDASI differ in terms of format. On the BPI, timeframe (recall period) is built into the question (e.g., pain at its worst in the last 24 hours), whereas the MDASI only provides the timeframe in the instructions (e.g., pain at its worst; instructions request patient to report "how severe the following symptoms have been in the past 24 hours"; p.61) [11]. Perhaps, patients did not read the full instructions and, as such, concluded their scoring represented overall ratings of severity. From this point, we discussed improvement of pain severity as reported on the BPI, in order to control for the ambiguity in recall period.

Pain severity both at its worst in the 24 hours prior to and at the time of BPI completion was significantly improved, independent of a relationship with time between first and second visit. Interestingly, average pain severity did not show a significant improvement but did decrease slightly in mean scores, suggesting average pain severity is not a quick linear decrease but rather, some days are better than others. At any rate, pain at its worst was improved indicating a decrease in severe pain events and providing the opportunity to engage in daily life activities.

\section{Symptom interference}

Our patient sample reported improved symptom interference on aspects of daily life, such as general activity, mood, and enjoyment of life, with a marginal decrease in symptom interference on their normal work and relationship with others. Patients recorded significant or marginal improvement in the MDASI affective sub-dimension (mood, enjoyment of life, and relationship with others). Mental health was improved as a product of engagement in integrated palliative care, confirming similar results from a previous study [7]. The MDASI activity sub-dimension (general activity, normal work, and walking) contained only one item associated with a significant relationship between first and second visit (general activity), while normal work presented as a marginally significant variable and walking appeared as non-significant. In terms of symptom interference within our population, results suggest affective aspects of daily life showed more improvement over activity aspects; although we cannot determine if mental health as a construct improves more quickly than physical health.

\section{Limitations}

Our data suggests the collaboration between the PHC and the Division of Palliative Medicine improved symptom burden among PHC patients infected with HIV. However, limitations do exist within our study. While paired-sample t-tests are theoretically able to manage small sample sizes [14], results for two MDASI items did not show statistical significance despite meeting the MDASI criteria for clinical significance (i.e., mean differences are greater than or equal to 0.98 1.21), suggesting a minimal loss of power within these items (i.e., MDASI 16 difference of means between first visit and second visit was 1.17 while MDASI 17 difference of means was 2.00) [11]. However, it is worth noting, MDASI 17 (interference of symptoms on relations with other people) did return a marginally significant result (i.e., $p=0.055$ ).

Our small sample size was driven by a low, survey-completion rate, such that only 27 (55\%) of the 49 patients referred to the program completed the BPI and MDASI at the initial visit. Due to the nature of our study (i.e., quality improvement and feasibility study), we did not collect data indicating a patient's reasoning for not completing the BPI or MDASI surveys. This outcome might reflect the relatively high rate of illiteracy, mental illness, and mistrust toward clinicians present in patients infected with HIV. Further, 38 (78\%) patients completed 
the instruments at the second assessment, possibly as a result of an established rapport with the nurse practitioner associated with their symptom improvement. Only 14 (28.57\%) patients met the inclusion criteria (i.e., completed the instruments at both visits).

\section{Conclusion}

We present this data as a building block to future studies. With this collaboration, our goal was to fill the gap within the literature and more importantly, provide a palliative program able to successfully address patients' comorbidities and symptom burden. Future studies will be needed to confirm our findings and determine the relative impact of the various components in a palliative care consult (e.g., medication management, the strength of the clinician-patient relationship, the time spend on education, etc.) on clinical outcomes. Additionally, it would be important to follow patients long-term to identify if their symptom improvement is maintained without undue side effects.

Although palliative care has traditionally been thought of as an end-of-life intervention, modern perspectives regard it as an adjuvant to general medical care. Palliative medicine programs embedded within outpatient, ambulatory HIV care clinics show potential to resolve the high symptom burden described by Merlin [2], Lofgren [15], and nearly every study in between.

Integrated palliative care programs are able to approach patients' concerns with chronic pain and other debilitating symptoms using a multidisciplinary approach. From this perspective, the patients' health is represented not as an isolated sector (e.g., chronic pain as a result of car accident) but as a product of diverse physical, psychological, social, and spiritual states (e.g., depression manifesting as a result of chronic pain interfering with aspects of daily life).

We believe our model of a palliative clinic, led specifically by an ACHPN, can further overcome barriers to effective palliative care for patients infected with HIV. Expertise and board certification in palliative medicine gives clinicians the skills and attitudes to address pain management needs and provide evidence-based treatment $[16,17]$. Currently, there are not enough physicians trained in palliative medicine to meet the needs of all patients with life-limiting illnesses, including HIV. The estimated gap between the supply and need for palliative medicine trained physicians may be as high as 18,000 [18] providers. While previous literature [19] found that providers can be effective when given proper education and access to resources (i.e., palliative medicine expert), physicians working in the U.S. are often unable to dedicate time to extensive training programs or workshops [20]. Nurse practitioners can fill the gap without sacrificing quality, as more time is available to devote to the patient [21]. In our study, patients were engaged in brief palliative care treatments through their PHC provider prior to referral to the ACHPN program. Despite receiving pain management care and medication during this period, patients reported high symptom burden at the initial visit with the ACHPN, confirming the claim that symptom burden remains prevalent in patients infected with HIV [2], despite provider engagement. Our results represent the select benefits of an integrated palliative care program over providerled pain management. Programs designed specifically to address pain management through expert palliative and hospice care hold the potential to eliminate barriers to effective palliative care and improve the prevalent, high symptom burden experienced by patients infected with HIV.

\section{References}

1. The Antimicrobial Therapy Cohort Collaboration (2008) Life expectancy of individuals on combination antiretroviral therapy in high-income countries: A collaborative analysis of 14 cohort studies. The Lancet. 372: 293-299.
2. Merlin JS, Cen L, Praestgaard A, Turner M, Obando A, et al. (2012) Pain and physical and psychological symptoms in ambulatory HIV patients in the current treatment era. Journal of Pain Symptom Management. 43: 638- 45.

3. Breitbart W, Kaim M, Rosenfeld B (1999) Clinicians' perceptions of barriers to pain management in AIDS. J Pain Symptom Manage 18: 203-212. [Crossref]

4. Harding R, Karus D, Easterbrook P, Raveis VH, Higginson IJ, et al. (2005) Does palliative care improves outcomes for patients with HIV/AIDS? A systematic review of the evidence. Sex Transm Infect 81: 5-14.[Crossref]

5. Selwyn PA, Forstein M (2003) Overcoming the false dichotomy of curative vs palliative care for late-stage HIV/AIDS: "let me live the way I want to live, until I can't". JAMA 290: 806-814.[Crossref]

6. Harding R, Simms V, Alexander C, Collins K, Combo E, et al. (2012) Can palliative care integrated within HIV outpatient settings improve pain and symptom control in a low-income country? A prospective, longitudinal, controlled intervention evaluation. AIDS Care: Psychological and Socio-medical Aspects of AIDS/HIV. 25: 795-804.

7. Lowther K, Selman L, Simms V, Gikaara N, Ahmed A, et al. (2015) Nurse-led palliative care for HIV-positive patients taking antiretroviral therapy in Kenya: A randomised controlled trial. The Lancet HIV. 2: e328-334.

8. Perry BA, Westfall AO, Molony E, Tucker R, Ritchie C, et al. (2013) Characteristics of an ambulatory palliative care clinic for HIV-infected patients. J Palliat Med 16: 934-937.[Crossref]

9. Simms V, Higginson IJ, Harding R (2012) Integration of palliative care throughout HIV disease. Lancet Infect Dis 12: 571-575.[Crossref]

10. http://www.mdanderson.org/education-and-research/departments-programs-andlabs/departments-and-divisions/symptom-research/symptom-assessment-tools/BPI UserGuide.pdf.

11. M.D. Anderson (2015b) M.D. Anderson's Brief Pain Inventory and Symptom Inventory Scale. Retrieved from http://www.mdanderson.org/education-and-research/ departments- Integrated palliative care program in HIV context 19 programs-andlabs/departments-and-divisions/symptomresearch/symptom-assessment-tools/MDASI userguide.pdf

12. Larue F, Fontaine A, Colleau SM (1997) Underestimation and undertreatment of pain in HIV disease: multicentre study. BMJ 314: 23-28.[Crossref]

13. De Cock KM, Mbori-Ngacha D, Marum E (2002) Shadow on the continent: public health and HIV/AIDS in Africa in the 21st century. Lancet 360: 67-72.[Crossref]

14. Zimmerman D (1997) Teacher's corner: A note on interpretation of the paired-samples $\mathrm{t}$ test. Journal of Educational and Behavioral Statistics. 22: 349-360.

15. Lofgren S, Friedman R, Ghermay R, George M, Pittman JR, et al. (2014) Integrating early palliative care for patients with HIV: Provider and patient perceptions of symptom and need for services. American Journal of Hospice Palliative Medicine.

16. Peretti-Watel P, Bendiane MK, Galinier A, Lapiana JM, Favre R, et al. (2004) Opinions toward pain management and palliative care: Comparison between HIV specialists and oncologists. AIDS Care. 16:619-627.

17. Fontaine A, Larue F, Lassaunière JM (1999) Physicians' recognition of the symptoms experienced by HIV patients: how reliable? J Pain Symptom Manage 18: 263-270. [Crossref]

18. LupuD, American Academy of Hospice , Palliative Medicine Workforce Task Force (2010) Estimate of current hospice and palliative medicine physician workforce shortage. Journal of Pain and Symptom Management. 40: 899-913.

19. Schuit K, Bender W, Meijler W, Otter R, Meyboom-Dejong B, et al. (1999). Learning effects of a workshop in palliative cancer care for general practitioners. Journal of Cancer Education. 14: 18-22.

20. Alexander C, Pappas G, Henley Y, Kangalawe A,Oyebola F, et al. (2015) Pain management for persons living with HIV disease: Experience with interprofessiona education in Nigeria. American Journal of Hospice and Palliative Medicine. 32: 555-562.

21. Heinle R, McNulty J, Hebert RS (2014) Nurse practitioners and the growth of palliative medicine. Am J HospPalliat Care 31: 287-291.[Crossref]

Copyright: (C)2016 McGraw SR. This is an open-access article distributed under the terms of the Creative Commons Attribution License, which permits unrestricted use, distribution, and reproduction in any medium, provided the original author and source are credited. 